\title{
A case report of critical ischemic stroke in moyamoya-like vasculopathy accompanied by systemic lupus erythematosus
}

\author{
Wooseong Jeong ${ }^{1}$, Gil Myeong Seong ${ }^{1}$, Jung-Hwan $\mathrm{Oh}^{2}$, Jay Chol Choi ${ }^{2}$, Joong-Goo Kim ${ }^{2}$ \\ ${ }^{1}$ Department of Internal Medicine, Jeju National University Hospital, Jeju National University School of Medicine, Jeju, Korea \\ ${ }^{2}$ Department of Neurology, Jeju National University Hospital, Jeju National University School of Medicine, Jeju, Korea
}

\begin{abstract}
Moyamoya-like vasculopathy (MMV) is a rare, chronic, progressive cerebrovascular disorder characterized by stenosis or occlusion of the terminal portion of the bilateral internal carotid arteries and development of abnormal collateral vessels at the base of the brain. This disorder develops in association with various systemic diseases and conditions, including neurofibromatosis type 1, Down syndrome, thyroid disease, radiation therapy, and autoimmune disease. We report a case of a 51-year-old female patient with low-activity systemic lupus erythematosus (SLE) who had a sudden onset of global aphasia and right hemiplegia. Three months previous, she had been on antiplatelet medication due to a single transient ischemic attack. Brain magnetic resonance imaging demonstrated a massive infarct of the left middle cerebral artery territory. Conventional angiography showed complete occlusion of the left middle cerebral artery with poor development of basal collateral vessels. This case demonstrates that a patient with underlying autoimmune disease such as SLE accompanied by MMV should be considered vulnerable to ischemic stroke.
\end{abstract}

Keywords: Moyamoya disease, Systemic lupus erythematosus, Stroke, Magnetic resonance imaging

\section{Introduction}

A large spectrum of inherited or acquired systemic diseases and conditions can occur in conjunction with progressive bilateral stenosis or occlusion of the major intracranial arteries, particularly within the circle of Willis. In addition, prominent development of pathological collateral circulation can be observed in the vicinity of steno-occlusive changes in these patients. This pathologic condition related to systemic conditions is known as moyamoya-like vasculopathy (MMV) [1]. MMV can develop in association with autoimmune disorders; several cohorts have reported a higher prevalence of autoimmune diseases in MMV than in the general population [2]. The clinical features and prognosis of MMV accompanied by autoimmune disease are unknown, and it is challenging to determine treatment in clinical practice. In this article, we re- port a female patient with systemic lupus erythematosus (SLE) who experienced a massive ischemic stroke a short time after a transient ischemic attack (TIA). We also analyzed high-resolution, 3-Tesla magnetic resonance vessel-wall imaging of the vessels before the occlusion.

\section{Case report}

A 51-year-old female patient visited the hospital with transient right hemiparesis and dysarthria. Initial magnetic resonance angiography (MRA) revealed severe narrowing of a distal segment of both internal carotid arteries and near occlusion at the M1 segment of a left middle cerebral artery (MCA), suggesting MMV (Figure 1A). High-resolution vessel-wall magnetic resonance imaging (MRI) revealed diffuse concentric vascular narrowing and absence of atherosclerotic plaque

Received: September 17, 2021 Revised: October 24, 2021 Accepted: November 1, 2021

Correspondence: Joong-Goo Kim

Department of Neurology, Jeju National University Hospital, Aran-13 gil, Jeju 63241, Korea

E-mail: lilis1118@naver.com

ORCID: https://orcid.org/0000-0002-4166-6023

Copyright (C) 2022 by The Korean Encephalitis and Neuroinflammation Society

This is an open access article distributed under the terms of the Creative Commons Attribution Non-Commercial License (http://creativecommons.org/licenses/by-nc/4.0/) which permits unrestricted non-commercial use, distribution, and reproduction in any medium, provided the original work is properly cited. 
Figure 1 Initial and follow-up neuroimaging of the patient
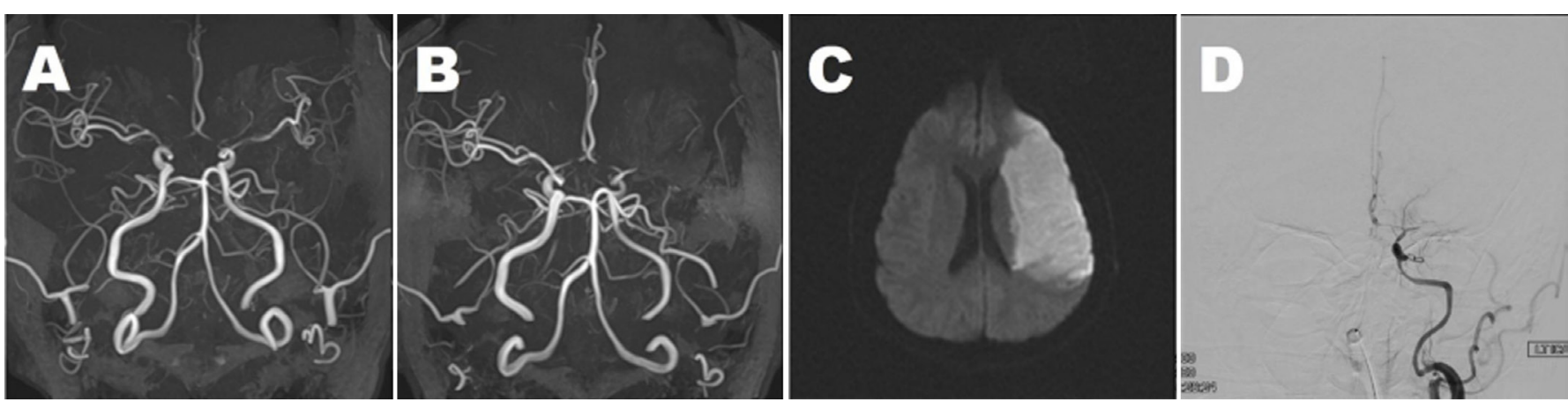

(A) Axial projected three-dimensional (3D) time-of-flight (TOF) magnetic resonance angiography (MRA) shows severe stenosis of both terminal internal carotid arteries, proximal arteries, middle cerebral arteries (MCAs), and the A1 segment of anterior cerebral arteries. (B) Three months later, axial projected 3D TOF MRA shows complete occlusion of the left MCA with M1 stump. (C) Diffusion-weighted image at the level of the lateral ventricle demonstrated massive acute infarctions in the left MCA territory. (D) Digital subtraction angiography shows occlusion of the proximal portion of the left MCA with minimal basal collateral vessel development.

without significant mural enhancement in the stenotic segment. Characteristics of the stenotic segment showed relatively concentric, nonremodeled lesions, without $\mathrm{T} 2$ wall high-signal intensity (Figure 2A-C). The patient also was diagnosed with SLE based on photosensitivity, thrombocytopenia, high titers of antinuclear antibody (ANA; 1:1,280) and anti-double-stranded DNA (anti-dsDNA) ( $80 \mathrm{IU} / \mathrm{mL})$, and decreased complement 3 (C3). Aspirin and a statin were administered after diagnosis of TIA associated with MMV. The RNF213 gene was negative. Evaluation for antiphospholipid syndrome, such as testing for lupus anticoagulant, anticardiolipin antibodies, and anti-beta2-glycoprotein antibodies, was negative. The SLE disease activity index score was 4 (mild activity), so we decided to follow up with hydroxychloroquine.

Three months later, the patient was admitted to our hospital with global aphasia and right hemiplegia with a power of $1 / 5$ in the upper and lower extremities after waking in the morning. MRI showed acute infarctions in the left MCA territory. MRA showed complete occlusion of the left MCA at the proximal M1 segment (Figure 1B, C). Conventional angiography demonstrated diffuse luminal narrowing in both internal carotid arteries (ICAs) and complete occlusion of the left MCA with minimal development of basal collateral vessels (Figure 1D).

Laboratory tests revealed a white blood cell count of 9,400 cells $/ \mathrm{mm}^{3}$ (reference, $4,000-10,000$ cells $/ \mathrm{mm}^{3}$ ), a hemoglobin level of $12.7 \mathrm{~g} / \mathrm{dL}$ (reference, 12-16 g/dL), a platelet count of 161,000 cells $/ \mathrm{mm}^{3}$ (reference, $150,000-450,000$ cells $/ \mathrm{mm}^{3}$ ), an erythrocyte sedimentation rate of $63 \mathrm{~mm} / \mathrm{hr}$ (reference, 0-20 $\mathrm{mm} / \mathrm{hr}$ ), a C-reactive protein level of $0.81 \mathrm{mg} / \mathrm{dL}$ (reference,
$0-0.3 \mathrm{mg} / \mathrm{dL}$ ), a serum protein level of $6.3 \mathrm{~g} / \mathrm{dL}$ (reference, 6.7-8.3 g/dL), a serum albumin level of $3.1 \mathrm{~g} / \mathrm{dL}$ (reference, $3.8-5.3 \mathrm{~g} / \mathrm{dL}$ ), a blood urea nitrogen level of $22.3 \mathrm{mg} / \mathrm{dL}$ (8-20 $\mathrm{mg} / \mathrm{dL}$ ), a serum creatinine level of $0.69 \mathrm{mg} / \mathrm{dL}$ (reference, $0.6-1.1 \mathrm{mg} / \mathrm{dL}$ ), a C3 level of $59 \mathrm{mg} / \mathrm{dL}$ (reference, $65-135 \mathrm{mg} /$ $\mathrm{dL}$ ), a C4 level of $13 \mathrm{mg} / \mathrm{dL}$ (reference, $13-35 \mathrm{mg} / \mathrm{dL}$ ), and a total complement activity level of $30.5 \mathrm{U} / \mathrm{mL}$ (reference, 23-46 $\mathrm{U} / \mathrm{mL}$ ). The patient's test results were negative for cytoplasmic-antineutrophil cytoplasmic autoantibody and perinuclear antineutrophil cytoplasmic autoantibody. The lipid profile showed a total cholesterol level of $115 \mathrm{mg} / \mathrm{dL}$ (reference, 130 $250 \mathrm{mg} / \mathrm{dL}$ ), a triglyceride level of $80 \mathrm{mg} / \mathrm{dL}$ (reference, 50 $130 \mathrm{mg} / \mathrm{dL}$ ), a high-density lipoprotein cholesterol level of 33 $\mathrm{mg} / \mathrm{dL}$ (reference, 33-83 mg/dL), and a low-density lipoprotein cholesterol level of $57 \mathrm{mg} / \mathrm{dL}$ (reference, $0-130 \mathrm{mg} / \mathrm{dL}$ ). Urinalysis showed 1+ proteinuria, microscopic hematuria (20-29 red blood cells/high-power field [HPF]), and microscopic pyuria (many white blood cells/HPF). An immunologic study showed ANA 1:1,280 (homogeneous pattern), anti-dsDNA $75 \mathrm{IU} / \mathrm{mL}$ (reference, 10-15 IU/mL), anti-Smith (-), anti-ribonucleoprotein (-), anti-Ro/anti-La (+/-), venereal disease research laboratory $(-)$, lupus anticoagulant (-), anti-cardiolipin IgG/IgM (-/-), and anti- $\beta 2$ glycoprotein I antibody (-). The anti-thrombin III level, prothrombin time, and activated partial thromboplastin time were within normal limits.

Fortunately, extensive territorial infarct did not advance to severe hemorrhagic transformation or brain swelling. Ninety days later, the patient's motor power in the upper and lower 
Figure 2 T2 vessel-wall magnetic resonance imaging
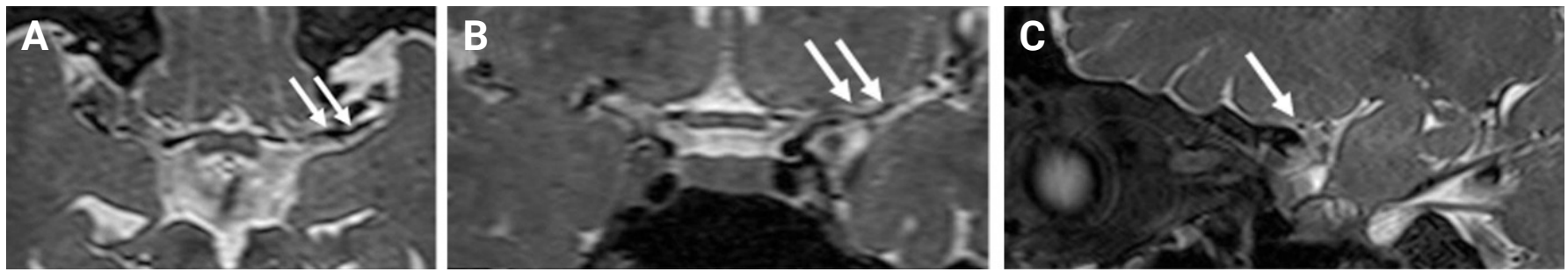

On axial (A), coronal (B), and sagittal (C) T2 vessel-wall magnetic resonance imaging at 3 months prior, the left middle cerebral arteries territory infarction shows severe stenosis without significant wall thickening or vascular remodeling proximal to the level of stenosis (white arrows).

extremities had improved to grade II, but there was no further improvement of language function (modified Rankin scale 4).

\section{Discussion}

This patient satisfied the Systemic Lupus International Collaborating Clinics criteria for a diagnosis of SLE, with a clinical spectrum disorder characterized by photosensitivity, thrombocytopenia, and an array of immunological abnormalities [3]. Cerebrovascular disease has been reported in $15 \%$ to $19 \%$ of patients with SLE $[4,5]$. The incidence ratio of stroke in patients with SLE was significantly higher than in the general population [5]. Among patients with SLE, baseline disease activity, hyperlipidemia, and hypertension are risk factors for cerebrovascular disease [5]. However, about $50 \%$ of cases of cerebrovascular disease in patients with SLE showed no evidence of increased disease activity [6]. About $40 \%$ of symptomatic large-vessel steno-occlusive disease in SLE resulted in poor outcomes [6], but there are few reports of an association between SLE and MMV.

The precise pathomechanism for MMV associated with SLE is unclear. In contrast to MMV, in vasculitis related to SLE, multiple vascular changes were observed in relatively small-diameter vessels. This case showed localized stenosis and characteristic development of collateral circulation around the circle of Willis. Thus, it can be assumed that the pathophysiology of our case is different from that caused by vasculitis related to SLE flare. Similar to our case, Kim et al. [7] reported high-resolution MRI findings that MMV was associated with smaller diameter and concentric stenosis compared with symptomatic atherosclerotic lesion. Most imaging patterns of acute ischemic stroke in patients with SLE and MMV were predominantly embolic type $[8,9]$. A characteristic finding in our case is an embolic pattern involving the whole territory despite ongoing stenosis. This pattern can be distinguished from the atherosclerotic occlusion pattern and is presumed to be due to rapid progression of steno-occlusion and poor development of collateral flow. Positivity for serum autoantibodies has been reported in patients with MMV, suggesting an autoimmune component in its pathogenesis [2]. Our case had no significant underlying vascular risk factors of cerebrovascular disease, such as high lupus activity, hypertension, diabetes mellitus, or hyperlipidemia. Thus, it was challenging to establish an optimal strategy for predicting and preventing disease progression. There was no evidence to support whether medical or surgical treatment would be effective for cerebrovascular disease in SLE-related MMV. In previous reports, immunosuppression indicates an effect for patients with high SLE activity, but proper drug selection and duration of treatment are unclear for MMV-related SLE [10,11]. Surgical management of MMV might prevent ischemia by restoring and enhancing cerebral blood flow [12]. However, several reports have shown successful disease control with only medical treatment [11]. There are no clear and definite criteria for surgical treatment in MMV. In our case, medical rather than surgical management was chosen because the patient experienced only one event of TIA and showed a stable clinical course for three months. Additionally, there was no significant evidence of SLE flares such as fever, erythema, arthritis, or laboratory abnormalities, and we could not properly assess SLE activity due to severe neurologic dysfunction. Also, vessel-wall evaluation was performed with high-resolution vessel-wall MRI when the patient first visited the hospital. Although previous studies have shown that high-resolution vessel-wall MRI can help differentiate the cause of vasculopathy $[13,14]$, it has not been used to evaluate disease activity or predict prognosis as shown in our case.

However, medical treatment for our patient failed, and fatal 
cerebral infarction with severe residual sequelae occurred three months after the first symptom. Although high-resolution MRI was used to evaluate the characteristics of intracranial stenosis diagnosed at the first TIA, it did not help predict progression to occlusion. Mikdash et al. [5] reported that stroke in SLE patients might be more severe than in non-SLE controls. In a prospective cohort study, about $77 \%$ of patients with stroke exhibited National Institutes of Health Stroke Scale $>6$. Additionally, individuals with SLE have a lower rate of survival after stroke compared to the general population [15].

In conclusion, it is important to recognize early progression in patients who have MMV with coexisting autoimmune diseases such as SLE. Surgical treatment should be considered for patients with MMV with lupus who had symptomatic eloquent location stenosis resulting in a critical impact on clinical outcome and quality of life.

\section{Conflicts of Interest}

No potential conflict of interest relevant to this article is reported.

\section{Author Contributions}

Conceptualization, Data curation, Investigation: Kim JG; Supervision: Choi JC, Kim JG; Validation: Choi JC; Writing-original draft: Jeong W, Kim JG; Writing-review \& editing: Seong GM, Oh JH, Kim JG.

\section{Acknowledgments}

This work was supported by a research grant from Jeju National University Hospital in 2018.

\section{References}

1. Suzuki J, Takaku A. Cerebrovascular "moyamoya" disease: disease showing abnormal net-like vessels in base of brain. Arch Neurol 1969;20:288-299.

2. Wanifuchi H, Kagawa M, Takeshita M, Izawa M, Maruyama S, Kitamura K. Autoimmune antibody in moyamoya disease. No Shinkei Geka
1986;14:31-35.

3. Petri M, Orbai AM, Alarcon GS, et al. Derivation and validation of the Systemic Lupus International Collaborating Clinics classification criteria for systemic lupus erythematosus. Arthritis Rheum 2012; 64:2677-2686.

4. Futrell N, Millikan C. Frequency, etiology, and prevention of stroke in patients with systemic lupus erythematosus. Stroke 1989;20:583-591.

5. Mikdashi J, Handwerger B, Langenberg P, Miller M, Kittner S. Baseline disease activity, hyperlipidemia, and hypertension are predictive factors for ischemic stroke and stroke severity in systemic lupus erythematosus. Stroke 2007;38:281-285.

6. Mitsias P, Levine SR. Large cerebral vessel occlusive disease in systemic lupus erythematosus. Neurology 1994;44(3 Pt 1):385-393.

7. Kim YJ, Lee DH, Kwon JY, et al. High resolution MRI difference between moyamoya disease and intracranial atherosclerosis. Eur J Neurol 2013;20:1311-1318.

8. Kim DY, Son JP, Yeon JY, et al. Infarct pattern and collateral status in adult moyamoya disease: a multimodal magnetic resonance imaging study. Stroke 2017;48:111-116.

9. Tsoi LK, Mok CC, Man BL, Fu YP. Imaging pattern and outcome of stroke in patients with systemic lupus erythematosus: a case-control study. J Rheumatol 2021;48:533-540.

10. Mok CC, Poon WL. Moyamoya phenomenon in systemic lupus erythematosus. Rheumatology (Oxford) 2011;50:1931.

11. Wang R, Xu Y, Lv R, Chen J. Systemic lupus erythematosus associated with Moyamoya syndrome: a case report and literature review. Lupus 2013;22:629-633.

12. El Ramahi KM, Al Rayes HM. Systemic lupus erythematosus associated with moyamoya syndrome. Lupus 2000;9:632-636.

13. Mossa-Basha M, de Havenon A, Becker KJ, et al. Added value of vessel wall magnetic resonance imaging in the differentiation of Moyamoya vasculopathies in a non-Asian cohort. Stroke 2016;47:1782-1788.

14. Muraoka S, Araki Y, Taoka T, et al. Prediction of intracranial arterial stenosis progression in patients with Moyamoya vasculopathy: contrast-enhanced high-resolution magnetic resonance vessel wall imaging. World Neurosurg 2018;116:e1114-e1121.

15. Yurkovich M, Vostretsova K, Chen W, Aviña-Zubieta JA. Overall and cause-specific mortality in patients with systemic lupus erythematosus: a meta-analysis of observational studies. Arthritis Care Res (Hoboken) 2014;66:608-616. 